\title{
Perception $v$. actual intakes of junk food and sugar-sweetened beverages in Australian young adults: assessed using the mobile food record
}

\author{
Amelia J Harray ${ }^{1}$, Carol J Boushey ${ }^{2}$, Christina M Pollard ${ }^{1,3}$, Chloe E Panizza ${ }^{2}$, \\ Edward J Delp ${ }^{4}$, Satvinder S Dhaliwal ${ }^{1}$ and Deborah A Kerr ${ }^{1, *}$ \\ ${ }^{1}$ School of Public Health, Curtin University, Bentley, Perth, WA 6102 Australia: ${ }^{2}$ Epidemiology Program, University \\ of Hawaii Cancer Center, Honolulu, HI, USA and Nutrition Program, Purdue University West Lafayette, IN, USA: \\ ${ }^{3}$ Department of Health in Western Australia, Perth, Australia: ${ }^{4}$ Video and Image Processing Laboratory, School of \\ Electrical and Computer Engineering, Purdue University, West Lafayette, IN, USA
}

Submitted 29 April 2016: Final revision received 1 March 2017: Accepted 15 March 2017: First published online 5 June 2017

\begin{abstract}
Objective: To determine perception $v$. actual intakes of energy-dense nutrient-poor 'junk food' (JF) and sugar-sweetened beverages (SSB) in young adults, using the mobile food record (mFR).

Design: Before-and-after eating images using a $4 \mathrm{~d} \mathrm{mFR}$ were assessed for standardised $600 \mathrm{~kJ}$ (143 kcal) servings of JF and SSB (excluding diet drinks). Participants reported their concern about the health aspects of their diet, perceptions and intentions regarding JF and SSB.

Setting: Perth, Western Australia.

Subjects: Adults ( $n$ 246) aged 18-30 years.

Results: The mean (SD) intake of JF + SSB was $3.7(2 \cdot 0)$ servings/d. Women thinking about drinking less SSB consumed more SSB servings/d (1.5 (1.2)) than men $(0.7$ $(0.5) ; P<0.05)$ who were thinking about drinking less. Men not thinking about cutting down JF consumed more servings/d (4.6 (2.4)) than women (2.5 (0.7); $P<0.01)$ who were not thinking about cutting down. Those who paid a lot of attention to the health aspects of their diet consumed less JF + SSB than those who took only a bit of notice $(P<0 \cdot 001)$, were not really thinking much about it $(P<0 \cdot 001)$ or who didn't think at all about the health aspects of food $(P<0 \cdot 01)$. Conclusions: Perceptions and attitudes regarding JF and SSB were associated with level of consumption. Those not thinking about cutting down their intake of these foods represent an important target group as they consume more than their peers. Further research is needed to identify how amenable young adults are to changing their intake, particularly given the lack of attention paid to the health aspects of their diet.
\end{abstract}

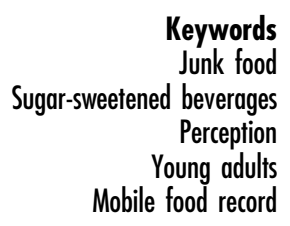

Worldwide, overweight and obesity rates are increasing and are related to significant health consequences ${ }^{(1)}$. Nearly half of young adults aged 18-34 years in Western Australia are classified as overweight or obese ${ }^{(2)}$. In Australia, 25-34-yearolds have the greatest annual increment in waist circumference and weight compared with any other age group and are gaining weight at a faster rate than in previous generations $^{(3)}$. To slow the trajectory of weight gain, this age group is an important target population for nutrition interventions that improve dietary habits. The excessive energy intake from the frequent consumption of 'energy-dense nutrient-poor' (EDNP) foods and sugar-sweetened beverages (SSB) and physical inactivity are modifiable risk factors associated with weight gain ${ }^{(4)}$. EDNP, commonly referred to as 'junk food' (JF) by the general public, are those foods and beverages high in energy, saturated fat, added sugar, salt or alcohol, and low in nutrients ${ }^{(5)}$.

The availability and affordability of EDNP foods and SSB worldwide have led to higher consumption of these foods and increased likelihood of excessive energy consumption $^{(6)}$. Regularly consuming an energy intake above energy requirements is associated with overweight, obesity, CVD, type 2 diabetes and some cancers ${ }^{(7,8)}$. Hence, the Australian Dietary Guidelines recommend limiting the frequency of consumption of these unnecessary EDNP foods and beverages (referred to as 'discretionary choices' in the 
Australian Dietary Guidelines) to 'occasionally' and in small amounts $^{(5)}$. Adherence to the Australian Dietary Guidelines in young adults is poor. In 2011/12, the Australian National Health Survey found that adults consumed approximately $35 \%$ of their total energy intake from EDNP foods and beverages, with young adults more likely to choose highly processed convenient options, such as hot chips, meat pies, chocolate, SSB, commercial burgers and pizzas ${ }^{(9,10)}$. Previous research highlights that consuming excess amounts of EDNP foods and SSB may be key factors in the rapid increase in body weight ${ }^{(9,11)}$.

Consumption of SSB has been identified as a key public health issue due to the frequency of consumption, low satiety, high added sugar content and associations with excess weight gain $^{(12)}$. Over half (53\%) of men and 39\% of women aged 19-30 years consume SSB on any given day in Australia ${ }^{(10)}$. A Western Australian study found that males with obesity and those aged 18-44 years were most likely to consume SSB ${ }^{(13)}$. Reducing EDNP foods and SSB are important targets for population-based interventions; therefore, exploring perceptions and attitudes towards these foods may lead to better outcomes. A study found a disconnect between perceived diet quality and whether dietary recommendations were being met, indicating the need to assess both dietary intake and perceptions ${ }^{(14)}$. Accurate assessment of dietary intake, however, is difficult.

While all dietary assessment methods are accompanied by a level of participant and researcher burden, collecting accurate dietary intake data from young adults is particularly challenging. As young adults are high users of mobile devices, technology-based dietary assessment methods incorporating digital or image-based recording may be more appealing ${ }^{(15,16)}$. An image-based method known as the mobile food record (mFR) app appears to have high acceptance among young people and shows potential for upscaling to population-wide nutrition monitoring ${ }^{(17,18)}$.

Several studies have identified the unrealistic perception of dietary fat, fruit and vegetable intakes as barriers for improving dietary intake ${ }^{(19-22)}$, highlighting a potential disconnect between what people think they are eating and what they are actually eating. Future interventions assessing both perceived intake (using questionnaires) and actual intake (using the mFR) could segment questions which more accurately represent actual intake. The current cross-sectional study aimed to assess whether young adults' perception of their current diet is associated with their intakes of JF and SSB, collected using a $4 \mathrm{~d}$ mFR.

\section{Methods}

\section{Study design}

This study was a cross-sectional analysis of the baseline data collected during a 6-month randomised controlled trial, the Connecting Health and Technology (CHAT) study $^{(17,23,24)}$. Participants were asked to attend Curtin University on two separate occasions, one week apart. During the initial visit, height and weight were recorded and participants were asked to complete a paper-based questionnaire to collect information on demographics and their knowledge and attitudes relating to food, nutrition and health. Participants were lent a mobile device (Apple iPod Touch) and trained how to use the specifically designed dietary assessment tool, the CHAT App, pre-uploaded on to the device. Participants were asked to collect an $\mathrm{mFR}$ for four consecutive days. Approval for the study was granted by the Curtin Human Ethics Research Committee and the trial was registered (Australian Clinical Trials Registry registration number ACTRN12612000250831)

\section{Participants}

Adults living in the Perth Metropolitan Area were recruited via the federal electoral roll. Screening occurred either online using a survey website or on the telephone to ensure the inclusion criteria were satisfied (18-30 years old and owned a mobile telephone). Potential participants were excluded if they: (i) were unable to attend on four occasions to complete the 6-month randomised controlled trial; (ii) studied nutrition; (iii) took part in extreme forms of exercise; (iv) followed a restrictive diet; or (v) were pregnant or breast-feeding.

\section{Data collection}

Participants completed a $4 \mathrm{~d} \mathrm{mFR}$ using an app running on an Apple iPod Touch. Details of the mFR CHAT App, also known as Technology Assisted Dietary Assessment system (TADA), and its use in dietary assessment have been described previously ${ }^{(18,25,26)}$. Participants were asked to take before-and-after eating images of all meals, snacks and beverages consumed over four consecutive days (Wednesday to Saturday). Participants were instructed to record any forgotten foods or beverages in the notes section of the iPod Touch or in the small paper booklet provided. On completion of the $\mathrm{mFR}$, the research dietitian clarified the contents of each image with participants in an open-ended approach, avoiding the use of leading questions. Where the contents of the images were unclear (e.g. food was covered up or lighting issues with image quality), the dietitian verified and recorded the image contents.

\section{Dietary analysis}

Post confirmation of the image contents, all food records were assessed by the research assistants for servings of JF and SSB, classified according to the Australian Guide to Healthy Eating standard definition of discretionary choices $^{(5)}$. Two research assistants trained in dietary assessment entered the food and beverage items into an 
electronic database independently. These data were checked by a third researcher (A.J.H.) who confirmed the content of the images and the portion size entered and corrected any discrepancies. One standard serving of 'discretionary food' including SSB was equivalent to $600 \mathrm{~kJ}$ ( $143 \mathrm{kcal})$; for example, twelve hot chips, one slice of commercial pizza, $25 \mathrm{~g}$ chocolate or $375 \mathrm{ml} \mathrm{SSB}$ (including cordials, soft drinks and flavoured mineral waters, energy and electrolyte drinks, and fruit drinks). Artificially sweetened beverages were reported separately and not included in the servings of SSB. Beverages already poured into glasses were classified by type (i.e. SSB) and the volume was estimated based on the size of the drinking vessel. Where the volume could not be confirmed by the participant, the research dietitian used the fiducial marker to estimate volume. Pure (100\%) fruit juices were classified according to the Australian Guide to Healthy Eating. The first $125 \mathrm{ml}$ ( $1 / 2$ cup) of $100 \%$ juice consumed was capped as one serving of fruit, then intake above this was counted as SSB servings. Although alcohol is also considered a discretionary food (due to it being energy-dense and nutrient-poor) and a contributor to overall energy intake, it was not included in the calculation of JF or SSB servings for the present study.

\section{Attitudes towards current dietary behaviours}

Written questionnaires collected demographics (age, ethnicity, employment status, income, living arrangements, education level, cooking abilities) and three questions measured attitudes regarding health aspects of diet and self-perception of current SSB and JF consumption. These questions were previously used in the Western Australian Nutrition Monitoring Survey Series ${ }^{(27)}$ and were selfcompleted by study participants on the first visit, prior to completing the mFR. The three outcome measures were:

1. Attention paid to the health aspects of diet were measured by asking 'Which statement best describes how you feel about your diet?', with response options of: (i) 'I pay a lot of attention to the health aspects of the food I eat to make sure my diet is as healthy as possible'; (ii) 'I take a bit of notice of the health aspects of the food I eat to make sure I have a fairly good diet'; (iii) 'I don't really think much about the health aspects of the food I eat'; (iv) and 'I don't think at all about the health aspects of the food I eat'.

2. Perception of current JF and SSB intakes was measured by the question 'Junk food or unhealthy food has been defined as food high in fat, sugar and/or salt with little nutritional value, such as fast food, crisps, sweetened breakfast cereals, confectionary or fizzy drinks. Which of the following best describes you?', with response options of: (i) 'I already eat a diet low in junk food'; (ii) 'I am currently trying to eat less junk food'; (iii) 'I am thinking about cutting down the amount of junk food I eat'; and (iv) 'I am not thinking about cutting down on the amount of junk food I eat'. The term 'junk food' was used as this is a commonly used contemporary term among Australians.

3. Intention towards changing current SSB intake was measured by asking 'Which of the following best describes you?' and the response options were: (i) 'I am currently trying to drink less sugary drinks (e.g. soft drinks, cordial, energy drinks or sports drinks)'; (ii) 'I am thinking about trying to drink less sugary drinks'; (iii) 'I am not thinking about cutting down on the amount of sugary drinks I have'; (iv) 'I already drink very little sugary drinks'; and (v) 'I don't drink sugary drinks'.

\section{Statistical analysis}

Descriptive statistics were used to assess the participants' physical characteristics and their mean intakes of JF and SSB servings (treated as a continuous variable). Comparison between the mean daily intakes of two groups (males $v$. females) was done using the two-sample $t$ test, and comparison between the four groups of participants was conducted using ANOVA. The perception of diet was compared with actual mean daily intakes of food group servings. ANOVA and $t$ tests were selected as we were comparing the group means for the daily intakes of JF and SSB servings collected over a $4 \mathrm{~d}$ mFR. One-way ANOVA was used to measure the differences between mean daily intake of JF or SSB servings using the mFR and how participants felt about their diet. Age was not significantly different between all groups compared $(P>0.05)$. The dependent variable for the analyses was the intake of JF only, SSB only, and JF and SSB combined $(\mathrm{JF}+\mathrm{SSB})$.

\section{Results}

\section{Demographics}

The study sample consisted of 247 participants (eighty-five men and 162 women), with a mean age of 24.3 (SD 3.4) years and a mean BMI of $24.3(\mathrm{SD} 5.4) \mathrm{kg} / \mathrm{m}^{2}$ (Table 1). One participant was excluded due to an incomplete $\mathrm{mFR}$ ( $n$ 246). Self-reported highest education level found that $36 \%$ of participants had completed school years ' 10,11 or 12 ', $24 \%$ had a trade or diploma, and $40 \%$ had a university degree or higher (see Table 1).

\section{Dietary intake of junk foods and sugar-sweetened beverages}

The mean intake of JF $+\mathrm{SSB}$ was $3 \cdot 7$ (SD 2.0) servings/d. Men consumed a mean of 3.3 (SD $2 \cdot 0$ ) servings of JF and 0.55 (SD 0.65) servings of SSB daily (Table 1). The mean intake for women was $3 \cdot 1$ (SD 1.4) servings JF/d and 0.46 (SD 0.59) servings SSB/d. There were no statistically significant differences between men and women for the 
Table 1 Participants' characteristics and actual mean intakes of junk food (JF) and sugar-sweetened beverages (SSB) over the $4 \mathrm{~d}$ mobile food record; young adults aged 18-30 years, Perth, Western Australia, Connecting Health and Technology study

\begin{tabular}{|c|c|c|c|c|c|c|}
\hline & \multicolumn{2}{|c|}{$\begin{array}{l}\text { Men } \\
(n 85)\end{array}$} & \multicolumn{2}{|c|}{$\begin{array}{l}\text { Women } \\
(n \text { 161) }\end{array}$} & \multicolumn{2}{|c|}{$\begin{array}{c}\text { Total sample } \\
(n \text { 246) }\end{array}$} \\
\hline & Mean & SD & Mean & SD & Mean & SD \\
\hline Age (years) & $24 \cdot 6$ & $3 \cdot 3$ & $24 \cdot 2$ & 3.4 & $24 \cdot 3$ & 3.4 \\
\hline Height $(\mathrm{m})$ & 1.78 & 0.1 & 1.65 & 0.1 & $1 \cdot 7$ & $0 \cdot 1$ \\
\hline Weight (kg) & $78 \cdot 7$ & $15 \cdot 1$ & $65 \cdot 6$ & $15 \cdot 6$ & $70 \cdot 1$ & $16 \cdot 6$ \\
\hline BMI $\left(\mathrm{kg} / \mathrm{m}^{2}\right)$ & $24 \cdot 7$ & 4.4 & $24 \cdot 1$ & $5 \cdot 8$ & $24 \cdot 3$ & $5 \cdot 4$ \\
\hline \multicolumn{7}{|l|}{ Dietary intake } \\
\hline $\mathrm{SSB}^{\star}$ & 0.5 & 0.6 & 0.5 & 0.6 & 0.5 & 0.6 \\
\hline $\mathrm{JF}^{\star}$ & 3.3 & $2 \cdot 0$ & $3 \cdot 1$ & 1.4 & 3.2 & 1.7 \\
\hline \multirow[t]{2}{*}{$\mathrm{JF}+\mathrm{SSB}$} & $3 \cdot 8$ & $2 \cdot 3$ & 3.6 & $1 \cdot 8$ & $3 \cdot 7$ & $2 \cdot 0$ \\
\hline & $n$ & $\%$ & $n$ & $\%$ & $n$ & $\%$ \\
\hline \multicolumn{7}{|l|}{ Education level } \\
\hline Year 10,11 or 12 & 32 & 38 & 56 & 35 & 88 & 36 \\
\hline Trade or diploma & 29 & 34 & 31 & 19 & 60 & 24 \\
\hline University degree or higher & 24 & 28 & 74 & 46 & 98 & 40 \\
\hline
\end{tabular}

*One serving of JF or SSB $=600 \mathrm{~kJ}$ (e.g. 1 serving $=375 \mathrm{ml} \mathrm{SSB}, 1$ serving $=25 \mathrm{~g}$ chocolate).

intakes of JF, SSB, or JF + SSB. Age and BMI $\left(<25 \cdot 0 \mathrm{~kg} / \mathrm{m}^{2}\right.$ or $\geq 25.0 \mathrm{~kg} / \mathrm{m}^{2}$ ) were not associated with intake of $\mathrm{JF}$, $\mathrm{SSB}$, or JF+SSB.

Of those who consumed SSB or artificially sweetened (diet) beverages, the most commonly consumed beverage was soft drink (e.g. cola, lemonade; $55 \%$ ), followed by fruit drink (25\%), diet drinks (15\%) and energy drinks $(3 \%)$. Although fruit drinks occupied a greater proportion of SSB consumed by women compared with men (see Fig. 1), there were no significant differences in the types of SSB consumed by gender.

\section{Perception of junk food intake compared with intake recorded using the mobile food record}

Perception of JF intake using the questionnaire and dietary intake assessed using the mFR are presented in Table 2. Participants who believed they were already eating a diet low in JF consumed a mean of 2.5 (SD 1.4) servings/d, less than those who reported currently trying to eat less JF (mean 3.3 (sD 1.5) servings/d; $P<0.001$ ), thinking about cutting down (mean 3.5 (sD 1.7) servings/d; $P<0.001$ ) or not thinking about cutting down (mean 3.8 (SD 2.2) servings $/ \mathrm{d} ; P<0 \cdot 001)$. Men who were not thinking about eating less JF consumed more per day than women who were also not thinking about eating less JF: mean 4.6 (SD 2.4) and 2.5 (SD 0.7) servings/d, respectively $(P<0.01$; see Table 2).

\section{Perception of sugary drink intake compared with intake recorded using the mobile food record}

Over $53 \%$ of participants reported drinking very little or no sugary drinks, $36 \%$ were either trying to drink less sugary drinks (27\%) or thinking about drinking less sugary drinks (9\%), and $11 \%$ were not thinking about cutting down on the amount of sugary drinks they consume (a)
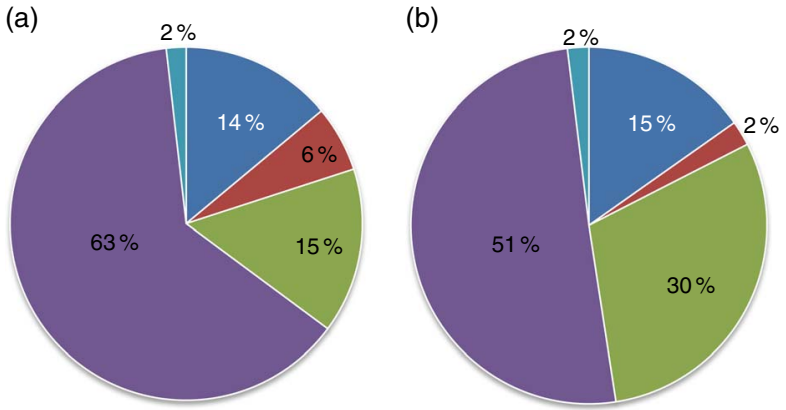

Fig. 1 (colour online) Types of sugar-sweetened beverages and artificially sweetened beverages consumed ( $\square$, diet drinks; $\square$, energy drinks; $\square$, fruit drinks; $\square$, soft drinks; $\square$, sports drinks), assessed using a $4 \mathrm{~d}$ mobile food record, by gender ( $a$, males; $b$, females); young adults (eighty-five men and 161 women) aged 18-30 years, Perth, Western Australia, Connecting Health and Technology study

(see Table 2). Among those thinking about drinking less sugary drinks, women consumed significantly more SSB than men (mean 1.5 (sD 1.2) and 0.7 (SD 0.5) servings/d, respectively; $P<0.05)$. However, in those not thinking about drinking less sugary drinks, men consumed significantly more SSB (mean 1.2 (SD 0.9) servings/d) than women (mean 0.5 (sD 0.4) servings/d; $P<0.05$ ).

One-way ANOVA revealed that participants who said they don't drink sugary drinks consumed less SSB than those who were trying to drink less sugary drinks $(0 \cdot 2 \mathrm{v}$. 0.6 servings $/ \mathrm{d}$, respectively; $P<0.05)$, thinking about drinking less sugary drinks $(1.0$ servings $/ \mathrm{d} ; P<0.001)$ and those not thinking about drinking less $(0.8$ servings/d; $P<0 \cdot 001$; see Table 2). Participants who said they already drink very little sugary drinks consumed less SSB than those who were trying to drink less $(P<0 \cdot 001)$, thinking about drinking less $(P<0.001)$ or were not thinking about 
Table 2 Perception of diet compared with actual mean daily intakes of junk foods (JF) and sugar-sweetened beverages (SSB) collected over the $4 \mathrm{~d}$ mobile food record (mFR), by gender; young adults aged 18-30 years, Perth, Western Australia, Connecting Health and Technology study

\begin{tabular}{|c|c|c|c|c|c|c|c|c|c|c|c|c|}
\hline \multirow[b]{3}{*}{ Perception of junk food intake } & \multicolumn{12}{|c|}{ Actual mean daily servings of JF over $4 \mathrm{~d} \mathrm{mFR}$} \\
\hline & \multicolumn{4}{|c|}{ Men $(n 84)$} & \multicolumn{4}{|c|}{ Women ( $n$ 158) } & \multicolumn{4}{|c|}{ Total sample ( $n$ 242) } \\
\hline & $n$ & $\%$ & Mean & $\mathrm{SD}$ & $n$ & $\%$ & Mean & SD & $n$ & $\%$ & Mean & SD \\
\hline 'I already eat a diet low in junk food' & 20 & 24 & $2 \cdot 3$ & 1.5 & 44 & 28 & $2 \cdot 6$ & 1.3 & 64 & 26 & 2.5 & 1.4 \\
\hline $\begin{array}{l}\text { 'I am currently trying to eat less junk } \\
\text { food' }\end{array}$ & 30 & 36 & $3 \cdot 1$ & 1.6 & 68 & 43 & 3.4 & 1.5 & 98 & 41 & $3 \cdot 3$ & 1.5 \\
\hline $\begin{array}{l}\text { 'I am thinking about cutting down the } \\
\text { amount of junk food I eat' }\end{array}$ & 19 & 22 & 3.6 & $2 \cdot 3$ & 38 & 24 & 3.5 & 1.4 & 57 & 23 & 3.5 & $1 \cdot 7$ \\
\hline \multirow{3}{*}{$\begin{array}{l}\text { 'I am not thinking about cutting down } \\
\text { on the amount of junk food I eat' }\end{array}$} & 15 & 18 & $4 \cdot 6^{\star \star}$ & $2 \cdot 4$ & 8 & 5 & $2 \cdot 5$ & 0.7 & 23 & 10 & $3 \cdot 8$ & $2 \cdot 2$ \\
\hline & \multicolumn{12}{|c|}{ Actual mean daily servings of SSB over $4 \mathrm{~d} \mathrm{mFR}$} \\
\hline & \multicolumn{4}{|c|}{ Men ( $n$ 85) } & \multicolumn{4}{|c|}{ Women $(n 160)$} & \multicolumn{4}{|c|}{ Total sample $(n$ 245) } \\
\hline Perception of sugary drink intake & $n$ & $\%$ & Mean & $\mathrm{SD}$ & $n$ & $\%$ & Mean & SD & $n$ & $\%$ & Mean & SD \\
\hline 'I don’t drink sugary drinks' & 4 & 5 & 0.1 & $0 \cdot 1$ & 13 & 8 & 0.3 & 0.6 & 17 & 7 & 0.2 & 0.5 \\
\hline $\begin{array}{l}\text { 'I already drink very little sugary } \\
\text { drinks' }\end{array}$ & 31 & 36 & 0.2 & 0.3 & 82 & 51 & 0.3 & 0.4 & 113 & 46 & 0.3 & 0.4 \\
\hline $\begin{array}{l}\text { 'I am currently trying to drink less } \\
\text { sugary drinks (soft drinks, cordial, } \\
\text { energy drinks or sports drinks)' }\end{array}$ & 24 & 28 & 0.6 & 0.7 & 42 & 26 & 0.6 & 0.5 & 66 & 27 & 0.6 & 0.6 \\
\hline $\begin{array}{l}\text { 'I am thinking about trying to drink less } \\
\text { sugary drinks' }\end{array}$ & 13 & 15 & $0.7^{*}$ & 0.5 & 9 & 6 & 1.5 & $1 \cdot 2$ & 22 & 9 & 1.0 & 0.9 \\
\hline \multirow{3}{*}{$\begin{array}{l}\text { 'I am not thinking about cutting down } \\
\text { on the amount of sugary drinks I have' }\end{array}$} & 13 & 15 & $1 \cdot 2^{*}$ & 0.9 & 14 & 9 & 0.5 & 0.4 & 27 & 11 & 0.8 & 0.7 \\
\hline & \multicolumn{12}{|c|}{ Actual mean daily servings of $\mathrm{JF}+\mathrm{SSB}$ over $4 \mathrm{~d} \mathrm{mFR}$} \\
\hline & \multicolumn{4}{|c|}{ Men ( $n$ 85) } & \multicolumn{4}{|c|}{ Women (n 159) } & \multicolumn{4}{|c|}{ Total sample ( $n$ 244) } \\
\hline Attention paid to health aspects of diet & $n$ & $\%$ & Mean & $\mathrm{SD}$ & $n$ & $\%$ & Mean & SD & $n$ & $\%$ & Mean & SD \\
\hline $\begin{array}{l}\text { 'I pay a lot of attention to the health } \\
\text { aspects of the food I eat to make sure } \\
\text { my diet is as healthy as possible' }\end{array}$ & 11 & 13 & $2 \cdot 2$ & 1.9 & 29 & 18 & $2 \cdot 8$ & 1.6 & 40 & 16 & $2 \cdot 6$ & $1 \cdot 7$ \\
\hline $\begin{array}{l}\text { 'I take a bit of notice of the health } \\
\text { aspects of the food I eat to make sure } \\
\text { I have a fairly good diet' }\end{array}$ & 50 & 59 & 3.9 & $2 \cdot 0$ & 97 & 60 & $3 \cdot 6$ & $1 \cdot 7$ & 147 & 60 & $3 \cdot 7$ & $1 \cdot 8$ \\
\hline $\begin{array}{l}\text { 'I don't really think much about the } \\
\text { health aspects of the food I eat' }\end{array}$ & 22 & 26 & $4 \cdot 4$ & $2 \cdot 7$ & 29 & 18 & $4 \cdot 2$ & $1 \cdot 8$ & 51 & 21 & $4 \cdot 3$ & $2 \cdot 2$ \\
\hline $\begin{array}{l}\text { 'I don't think at all about the health } \\
\text { aspects of the food I eat' }\end{array}$ & 2 & 2 & $6 \cdot 1$ & 0.3 & 4 & 3 & 4.2 & $2 \cdot 2$ & 6 & 3 & 4.8 & $2 \cdot 0$ \\
\hline
\end{tabular}

Significant differences between men and women (dependent variable: JF or SSB intake): ${ }^{*} P<0.05,{ }^{* *} P<0.01$.

drinking less $(P<0 \cdot 001)$. Participants trying to drink less sugary drinks consumed less SSB than those who were thinking about drinking less sugary drinks $(P<0.001)$ and those not thinking about drinking less $(P<0 \cdot 05)$.

\section{Attention paid to the bealth aspects of food eaten}

The level of attention participants paid to the health aspects of the food they ate was associated with their intakes of JF, $\mathrm{SSB}$, and $\mathrm{JF}+\mathrm{SSB}$ assessed using the $\mathrm{mFR}$ (Fig. 2). Those who paid a lot of attention consumed less JF + SSB than those who were taking a bit of notice $(P<0 \cdot 001)$, not thinking much about it $(P<0.001)$ or not thinking at all about it $(P<0 \cdot 01)$. Those who paid a lot of attention to the health aspects of the food they ate consumed a mean of $0 \cdot 25$ (SD 0.4) servings SSB/d, less compared with those who didn't really think much (mean 0.6 (SD 0.8 ) servings/d;
$P<0.05$ ) and those who didn't think at all (mean 1.4 (SD 0.8) servings/d; $P<0 \cdot 001)$. There was no significant difference in SSB intake between participants who paid a lot of attention and those who took only a bit of notice. Age, gender and BMI were not associated with attention participants paid to the health aspects of the food they ate.

\section{Discussion}

Participants' perceptions of their current JF and sugary drink intakes were associated with their intakes of these foods assessed using the mFR. There were significant associations between perceived and actual intakes of EDNP 'junk foods' (JF, SSB, JF + SSB) found in our study, highlighting the need to incorporate assessment 


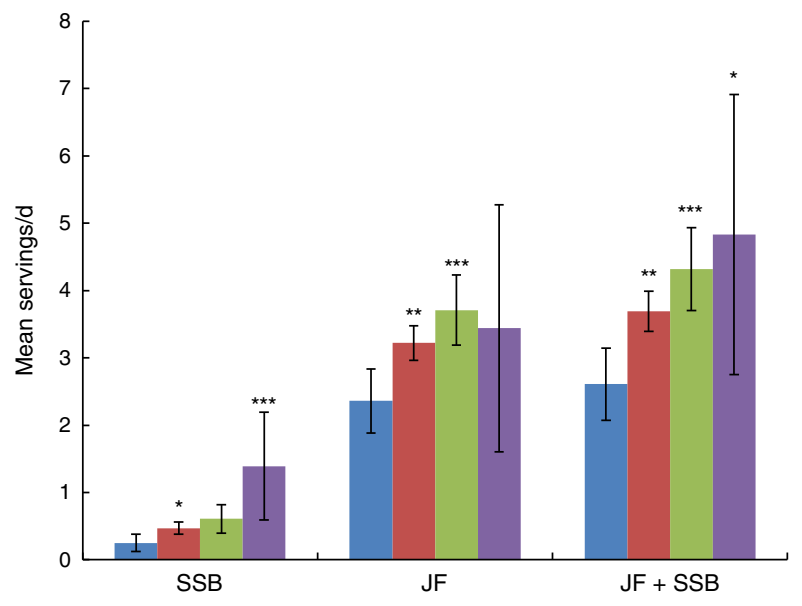

Fig. 2 (colour online) Associations between the level of attention participants paid to the health aspects of their diet ( $\square$, 'I pay a lot of attention to the health aspects of the food I eat'; $\square$, 'I take a bit of notice of the health aspects of the food I eat'; $\square$, 'I don't really think much about the health aspects of the food I eat'; $\square$, 'I don't think at all about the health aspects of the food I eat') and their actual mean daily intakes of junk foods (JF) and sugar-sweetened beverages (SSB) assessed using a $4 \mathrm{~d}$ mobile food record; young adults (eighty-five men and 161 women) aged 18-30 years, Perth, Western Australia, Connecting Health and Technology study. Values are mean servings per day ( 1 serving of JF or $S S B=600 \mathrm{~kJ}$; e.g. 1 serving $=375 \mathrm{ml} \mathrm{SSB}, 1$ serving $=25 \mathrm{~g}$ chocolate) with their standard deviations represented by vertical bars. Level of statistical significance using one-way ANOVA against 'I pay a lot of attention to the health aspects of the food I eat': ${ }^{*} P<0.05$, ${ }^{\star *} P<0.01,{ }^{* \star} P<0.001$

methods to strengthen knowledge regarding these dietary behaviours. Young adults who reported they were not thinking much or at all about the health aspects of the food they ate consumed significantly more JF + SSB than those who said they paid a lot of attention ( $4 \cdot 8 v .4 \cdot 2$ servings/d, respectively). Based on their consumption, they represent a priority target population. The challenge, however, is that they may be less amenable to changing their dietary behaviour as health is not a salient issue.

Young adults who perceived their diet to be low in JF consumed significantly less than those who felt their diets were high in JF; although they still consumed about 2.8 servings of $\mathrm{JF}+\mathrm{SSB}$ each day, equivalent to approximately $1700 \mathrm{~kJ}$ ( $406 \mathrm{kcal}$ ). The findings indicate that compared with their peers, young adults who believed they were already consuming a diet low in JF had a semi-accurate perception of their intake compared with the intake of their peers.

The majority of participants in the current study reported they were currently trying to eat less JF (40\%) or already eating a diet low in JF (26\%), indicating either a particularly motivated group or a strong influence of social desirability. Social desirability is evident when positive responses are given in the hope to impress the research investigators, either intentionally or unintentionally ${ }^{(28)}$, and can be a result of a person's knowledge of dietary recommendations or what he/she believes to be healthy or unhealthy dietary choices. Participants' modification of their dietary intake, for reasons of social desirability, is a potential influencing factor in all forms of self-reported dietary assessment, with JF more likely to be underreported than more nutritious foods ${ }^{(29-31)}$. In the current study, the mean intake of SSB was 0.5 servings/d (equivalent to $187 \mathrm{ml}$ ). These findings are consistent with another study in young Australian adults ${ }^{(32)}$. There, dietary intake was collected using a $5 \mathrm{~d}$ electronic dietary record and a median intake of $172 \mathrm{~g} \mathrm{SSB} / \mathrm{d}$ was found. However, we cannot rule out that although participants in the current study could not review or edit the mFR images, they may have altered their intakes of JF and SSB prior to capturing them. The recording process itself may have also raised the participants' awareness, leading to changes in food and beverage intakes. In addition, the Western Australian state government-funded LiveLighter ${ }^{\circledR}$ public health social marketing campaign ${ }^{(33,34)}$, which used mass media to discourage the consumption of JF and SSB, was being conducted around the time of data collection and therefore may have influenced participants' perceptions and dietary intakes.

The highest consumers of JF were men who reported they were not thinking about cutting down their JF intake. Almost one in five young men (18\%) consumed an average of 5.3 servings $\mathrm{JF}+\mathrm{SSB} / \mathrm{d}$, equivalent to approximately $3180 \mathrm{~kJ}(760 \mathrm{kcal})$ and higher than all other groups. This is despite the Australian Guide to Healthy Eating recommendations that these foods should be consumed only 'occasionally and in small amounts for good health'(35).

These findings indicate nutrition interventions may need to specifically target young men to increase awareness of their JF and SSB intakes. As with other behaviours, these findings also suggest nutrition messages regarding JF and SSB need to be targeted to participants' knowledge, attitudes, perceptions and behaviour, while acknowledging the need for healthier options to be available and accessible within their food environment ${ }^{(14,36)}$.

These findings reinforce the need to better understand the relationship between perceived diet and actual dietary intake as potential barriers to dietary change as this may lead to more appropriate targeting of nutrition messages and public health interventions. Powell-Wiley et al. ${ }^{(14)}$ investigated the association between perceived and objective diet quality using the Dietary Approaches to Stop Hypertension (DASH) index score, finding that among most ethnic groups those who perceived their diet quality to be higher received a higher objective diet quality index score. However, consistent with the findings of the current study, measured diet quality was not consistent with dietary recommendations.

The notable strengths of our study include the use of a population-based sample of young adults and the collection of dietary intake data over four consecutive days using the 
mFR app, including weekdays and a weekend day. Asking participants to report on how they perceived their diet prior to informing them of which elements of diet were being assessed strengthens these findings. Using the $\mathrm{mFR}$ to measure dietary intake provides a more objective method compared with short screening questions due to the level of systematic error inherent in methods whereby people are expected to estimate usual dietary intake ${ }^{(37)}$.

There are some limitations that need to be considered when interpreting the results of our study. First, a definition of the term 'junk food' was provided to participants with examples when completing the questionnaire; however, this was not a complete list. The foods classified as 'discretionary choices' in the Australian Guide to Healthy Eating for the analyses may not have been perceived as 'junk food or SSB' by some participants when answering the question, such as butter, mayonnaise or fruit juice drink. An understanding and sound knowledge of food, nutrition and dietary recommendations would be required to provide an accurate assessment of discretionary intake. Second, misreporting of JF and SSB may have occurred as a result of participants either modifying their usual dietary intake during the $4 \mathrm{~d} \mathrm{mFR}$ or being selective in the foods and beverages they took images of. Participants were asked to take images of all foods and beverages consumed over $4 \mathrm{~d}$ but only JF and SSB were considered in the present analyses. While a longer recording period may better capture the intake of JF and SSB, it tends to lead to less compliance. We elected to use $4 \mathrm{~d}$ as this is the typical recording period with paper-based food records and were mindful of participant burden, but this may not have fully captured usual intake. Lastly, although we attempted to recruit a population-based sample by recruiting through the federal electoral roll (a compulsory register of voters in Australia), it is possible that the findings are not representative of the population of young adults.

Previous research has highlighted a need to explore determinants of food behaviours in young adults as they transition from adolescence to adulthood ${ }^{(38)}$. Although there is a strong evidence base supporting the relationship between JF and SSB intakes and negative health outcomes, there is limited evidence on how young adults perceive their intakes, their intention to change and whether this is a determinant of food choice.

\section{Conclusions}

Young adults who pay more attention to the health aspects of food consume less JF and SSB, regardless of age, gender or BMI. Compared with their peers, young adults who believed they were already consuming a diet low in JF had a semi-accurate perception of their intake compared with the intake of their peers. However, even this group consumed unhealthy foods in excess of dietary recommendations for good health. The findings from the current cross-sectional analysis indicate the importance of assessing attitudes when developing nutrition interventions. Specific strategies are required for those who are not currently thinking about cutting down their intakes of JF and SSB and those who pay little or no attention to the health aspects of the food they eat.

\section{Acknowledgements}

Acknowledgements: The authors are grateful to the TADA project team, Marc Bosch, Ziad Ahmad and Maggie Zhu, for their technical support for the mobile food record and Aqif Mukhtar for the design of the Access database for the assessment of food groups. Financial support: This study was funded by a Healthway Health Promotion Research Grant with funding support also from the Department of Health, Western Australia and the National Institutes of Health/National Cancer Institute (grant number 1U01CA130784-01); and the National Institutes of Health/ National Institute of Diabetes and Digestive and Kidney Diseases (grant numbers 1R01-DK073711-01A1 and 2R56DK073711-04) for the mobile record. The funders had no role in the design, analysis or writing of this article. Conflict of interest: The authors of this manuscript have no conflicts of interest to declare. Authorship: A.J.H. formulated the research question, designed the study, carried out the study, analysed the data, and wrote and reviewed the manuscript. D.A.K. assisted in formulating the research question, assisted in developing the mobile food record, designed the study, carried out the study and reviewed the manuscript. C.J.B. assisted in formulating the research question, assisted in developing the mobile food record, helped design the study, assisted in data analysis and reviewed the manuscript. S.S.D. assisted in data analysis and reviewed the manuscript. C.M.P. assisted in formulating the research question and reviewed the manuscript. C.E.P. reviewed the manuscript. E.J.D. developed the mobile food record application and reviewed the manuscript. Ethics of human subject participation: This study was approved by the Curtin Human Ethics Research Committee and the trial was registered (Australian Clinical Trials Registry registration number ACTRN12612000250831).

\section{References}

1. World Health Organization (2015) Obesity and overweight. http://www.who.int/mediacentre/factsheets/ fs311/en/ (accessed April 2016).

2. Australian Bureau of Statistics (2016) 4364.0.55.001 - National Health Survey: First Results, 2014-15. Canberra: ABS.

3. Tanamas SK, Shaw JE, Backholer K et al. (2014) Twelve-year weight change, waist circumference change and incident obesity: the Australian diabetes, obesity and lifestyle study. Obesity (Silver Spring) 22, $1538-1545$. 
4. Australian Institute of Health and Welfare (2004) Risk Factor Monitoring. Canberra: Australian Government.

5. National Health and Medical Research Council (2013) Eat for Health: Australian Dietary Guidelines. Canberra: NHMRC.

6. Drewnowski A (2004) Obesity and the food environment: dietary energy density and diet costs. Am J Prev Med 27, 154-162.

7. Vartanian LR, Schwartz MB \& Brownell KD (2007) Effects of soft drink consumption on nutrition and health: a systematic review and meta-analysis. Am J Public Health 97, 667-675.

8. Martin-Calvo N, Martínez-González MA, Bes-Rastrollo M et al. (2014) Sugar-sweetened carbonated beverage consumption and childhood/adolescent obesity: a case-control study. Public Health Nutr 17, 2185-2193.

9. Rangan AM, Schindeler S, Hector DJ et al. (2009) Consumption of 'extra' foods by Australian adults: types, quantities and contribution to energy and nutrient intakes. Eur J Clin Nutr 63, 865-871.

10. Australian Bureau of Statistics (2012) 4364.0.55.007 Australian Health Survey: Nutrition First Results - Foods and Nutrients, 2011-12. Canberra: ABS.

11. Bes-Rastrollo M, Sanchez-Villegas A, Gomez-Gracia E et al. (2006) Predictors of weight gain in a Mediterranean cohort: the Seguimiento Universidad de Navarra Study. Am J Clin Nutr 83, 362-370.

12. Malik VS, Popkin BM, Bray GA et al. (2010) Sugar-sweetened beverages and risk of metabolic syndrome and type 2 diabetes: a meta-analysis. Diabetes Care 33, 2477-2483.

13. Pollard CM, Meng X, Hendrie GA et al. (2015) Obesity, socio-demographic and attitudinal factors associated with sugar-sweetened beverage consumption: Australian evidence. Aust N Z J Public Health 40, 71-77.

14. Powell-Wiley TM, Miller PE, Agyemang P et al. (2014) Perceived and objective diet quality in US adults: a cross-sectional analysis of the National Health and Nutrition Examination Survey (NHANES). Public Health Nutr 17, 2641-2649.

15. Boushey CJ, Harray AJ, Kerr DA et al. (2015) How willing are adolescents to record their dietary intake? The mobile food record. JMIR Mhealth Uhealth 3, e47.

16. Boushey CJ, Kerr DA, Wright J et al. (2009) Use of technology in children's dietary assessment. Eur J Clin Nutr 63, Suppl. 1, S50-S57.

17. Kerr DA, Harray AJ, Pollard CM et al. (2016) The connecting health and technology study: a 6-month randomized controlled trial to improve nutrition behaviours using a mobile food record and text messaging support in young adults. Int J Behav Nutr Phys Act 13, 52 .

18. Zhu F, Mariappan A, Boushey CJ et al. (2008) Technologyassisted dietary assessment. Proc SPIE Int SOC Opt Eng 6814, 681411 .

19. Brug J, van Assema P, Kok G et al. (1994) Self-rated dietary fat intake: association with objective assessment of fat, psychosocial factors, and intention to change. J Nutr Educ 26, 218-223.

20. Lechner L, Brug J \& De Vries H (1997) Misconceptions of fruit and vegetable consumption: differences between objective and subjective estimation of intake. J Nutr Educ 29, 313-320.

21. Bogers RP, Brug J, van Assema P et al. (2004) Explaining fruit and vegetable consumption: the theory of planned behaviour and misconception of personal intake levels. Appetite 42, 157-166.

22. Pollard CM, Daly AM \& Binns CW (2009) Consumer perceptions of fruit and vegetables serving sizes. Public Health Nutr 12, 637-643.

23. Kerr DA, Pollard CM, Howat P et al. (2012) Connecting Health and Technology (CHAT): protocol of a randomized controlled trial to improve nutrition behaviours using mobile devices and tailored text messaging in young adults. BMC Public Health 12, 477.

24. Harray AJ, Boushey CJ, Pollard CM et al. (2015) A novel dietary assessment method to measure a healthy and sustainable diet using the mobile food record: protocol and methodology. Nutrients 7, 5375-5395.

25. Zhu F, Bosch M, Khanna N et al. (2015) Multiple hypotheses image segmentation and classification with application to dietary assessment. IEEE J Biomed Health Inform 19, 377-388.

26. Zhu F, Bosch M, Woo I et al. (2010) The use of mobile devices in aiding dietary assessment and evaluation. IEEE J Sel Top Signal Process 4, 756-766.

27. Pollard CM, Harray AJ, Daly A et al. (2015) Nutrition Monitoring Survey Series 2012 Key Findings. Perth: Department of Health, Western Australia.

28. Cadmus-Bertram L \& Patterson RE (2013) Overview of nutritional epidemiology. In Nutrition in the Prevention and Treatment of Disease, 3rd ed., pp. 107-124 [AM Coulston, CJ Boushey and MG Ferruzzi, editors]. San Diego, CA: Academic Press.

29. Krebs-Smith SM, Graubard BI, Kahle LL et al. (2000) Low energy reporters vs others: a comparison of reported food intakes. Eur J Clin Nutr 54, 281-287.

30. Bingham SA, Cassidy A, Cole TJ et al. (1995) Validation of weighed records and other methods of dietary assessment using the $24 \mathrm{~h}$ urine nitrogen technique and other biological markers. Br J Nutr 73, 531-550.

31. Subar AF, Freedman LS, Tooze JA et al. (2015) Addressing current criticism regarding the value of self-report dietary data. J Nutr 145, 2639-2645.

32. Rangan AM, Tieleman L, Louie JC et al. (2016) Electronic Dietary Intake Assessment (e-DIA): relative validity of a mobile phone application to measure intake of food groups. Br J Nutr 115, 2219-2226.

33. Morley B, Niven P, Dixon H et al. (2016) Population-based evaluation of the 'LiveLighter' healthy weight and lifestyle mass media campaign. Health Educ Res 31, 121-135.

34. LiveLighter (2015) LiveLighter background. https:// livelighter.com.au/About/Background (accessed April 2016).

35. National Health and Medical Research Council (2003) Dietary Guidelines for Australian Adults. Canberra: Commonwealth of Australia.

36. Fishbein M \& Ajzen I (1975) Belief, Attitude, Intention, and Behavior: An Introduction to Theory and Research. Reading: MA: Addison-Wesley.

37. Kirkpatrick SI, Reedy J, Butler EN et al. (2014) Dietary assessment in food environment research: a systematic review. Am J Prev Med 46, 94-102.

38. Thorpe MG, Kestin M, Riddell LJ et al. (2014) Diet quality in young adults and its association with food-related behaviours. Public Health Nutr 17, 1767-1775. 